\title{
Silicon-organic hybrid devices
}

\author{
L. Alloatti ${ }^{\mathrm{a}, \mathrm{b}, *}$, D. Korn ${ }^{\mathrm{a}}$, J. Pfeifle ${ }^{\mathrm{a}}$, R. Palmer ${ }^{\mathrm{a}}$, S. Koeber ${ }^{\mathrm{a}}$, M. Baier ${ }^{\mathrm{a}}$, R. Schmogrow ${ }^{\mathrm{a}}$,

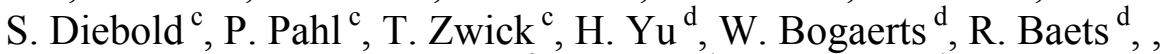 \\ M. Fournier ${ }^{\mathrm{e}}$, J. Fedelie, R. Dinu ${ }^{\mathrm{f}}$, C. Koos ${ }^{\mathrm{a}, \mathrm{b}}$, W. Freude ${ }^{\mathrm{a}, \mathrm{b}}$, J. Leuthold ${ }^{\mathrm{a}, \mathrm{b}}$ \\ ${ }^{a}$ Institute of Photonics and Quantum Electronics (IPQ), Karlsruhe Institute of Technology (KIT), \\ Engesserstr. 5, 76131 Karlsruhe Germany \\ ${ }^{\mathrm{b}}$ Institute of Microstructure Technology (IMT), Karlsruhe Institute of Technology (KIT), \\ Hermann-von-Helmholtz-Platz 1, 76344 Eggenstein-Leopoldshafen, Germany \\ ${ }^{\mathrm{c}}$ Institut fuer Hochfrequenztechnik und Elektronik (IHE), Karlsruhe Institute of Technology (KIT), \\ Kaiserstrasse 12, 76131 Karlsruhe Germany \\ ${ }^{\mathrm{d}}$ Photonics Research Group, Ghent University - IMEC, Department of Information Technology, \\ 9000 Gent, Belgium \\ ${ }^{\mathrm{e}} \mathrm{CEA}$, LETI, Minatec 17 rue des Martyrs, 38054 Grenoble, France \\ ${ }^{\mathrm{f}}$ GigOptix Inc., Switzerland and GigOptix Bothell (WA), USA \\ *luca.alloatti@kit.edu
}

\begin{abstract}
Silicon-organic hybrid (SOH) devices combine silicon waveguides with a number of specialized materials, ranging from third-order optically-nonlinear molecules to second-order nonlinear polymers and liquid-crystals. Second-order nonlinear materials allow building high-speed and low-voltage electro-optic modulators, which are key components for future silicon-based photonics transceivers. We report on a $90 \mathrm{GHz}$ bandwidth phase modulator, and on a $56 \mathrm{Gbit} / \mathrm{s}$ QPSK experiment using an IQ Pockels effect modulator. By using liquid-crystal claddings instead, we show experimentally that phase shifters with record-low power consumption and ultra-low voltage-length product of $V_{\pi} L=0.06 \mathrm{Vmm}$. Secondorder nonlinear materials, moreover, allow creating nonlinear waveguides for sum- or difference-frequency generation, and for lowest-noise optical parametric amplification. These processes are exploited for a large variety of applications, like in the emerging field of on-chip generation of mid-IR wavelengths, where pump powers are significantly smaller compared to equivalent devices using third-order nonlinear materials. In this work, we present the first SOH waveguide design suited for second-order nonlinear processes. We predict for our device an amplification of $14 \mathrm{~dB} / \mathrm{cm}$ assuming a conservative $\chi^{(2)}$-nonlinearity of $230 \mathrm{pm} / \mathrm{V}$ and a $\mathrm{CW}$ pump power as low as $20 \mathrm{dBm}$.
\end{abstract}

Keywords: Silicon-organic hybrid, silicon-on-insulator, electro-optic modulator, phase shifter, liquid-crystal, mid-IR generation, difference-frequency generation (DFG), second-order nonlinear waveguide.

\section{INTRODUCTION}

Silicon has several exceptional physical properties which make it one of the best materials for future low-power, lowcost and high-volume photonic integrated circuits (PICs) [1]. However, silicon suffers also from some fundamental limitations. For example, it has no second-order nonlinearity, has a large two-photon-absorption (TPA) coefficient, and is an indirect band gap semiconductor. As a consequence, silicon alone cannot be used for building Pockels effect modulators, cannot be pumped with high optical powers as often required in nonlinear processes, and cannot be exploited for light emission. By combining specialized cladding materials with silicon waveguides, it is possible to take advantage of the strong optical confinement and of the inexpensive silicon fabrication technology, while high optical nonlinearities, small TPA coefficients, voltage-dependent birefringence and optical emission come from tailored organic cladding materials. And indeed, high-speed modulators [2, 3], wavelength converters [4], low-power phase shifters [5, 6] and even lasers have been successfully demonstrated on the versatile silicon-organic hybrid ( $\mathrm{SOH}$ ) platform [7].

In this paper we review our recent advances in SOH high-speed modulators, showing the first silicon device having a $3 \mathrm{~dB}$ bandwidth higher than $90 \mathrm{GHz}$ and demonstrating high-speed data transmission with an IQ modulator. In addition,

Silicon Photonics VIII, edited by Joel Kubby, Graham T. Reed, Proc. of SPIE Vol. 8629, 86290P · @ 2013 SPIE · CCC code: 0277-786X/13/\$18 · doi: 10.1117/12.2005866 
highly-efficient liquid crystal phase shifters are reported. Finally, we propose a waveguide structure for achieving efficient difference-frequency generation.

\section{HIGH-SPEED SOH MODULATORS}

Optical modulators are key components for a variety of applications such as photonic transceivers or radio-over-fiber links. However, achieving low modulation voltage and high-speed operation still remains a challenge. Since silicon does not possess a $\chi^{(2)}$-nonlinearity, state-of-the art silicon photonic modulators mainly rely on free-carrier dispersion in pin or $p n$ junctions [8-10]. The latter concept enables higher modulation bandwidths, and $50 \mathrm{Gbit} / \mathrm{s}$ have already been achieved by on-off keying $(\mathrm{OOK})$ with a voltage-length product $V_{\pi} L=28 \mathrm{~V} \mathrm{~mm} \mathrm{[11].} \mathrm{Unfortunately,} \mathrm{such} \mathrm{plasma} \mathrm{effect} \mathrm{phase}$ modulators produce undesired intensity modulation as well, and they respond nonlinearly to the applied voltage. The hybrid integration of III-V epitaxy stacks grown in InP substrates and subsequently transferred to SOI waveguides is an alternative approach for creating high-speed electro-absorption modulators [12]. Recently, such a device demonstrated a $3 \mathrm{~dB}$ bandwidth larger than $67 \mathrm{GHz}$, becoming the fastest modulator realized so far on a silicon chip.

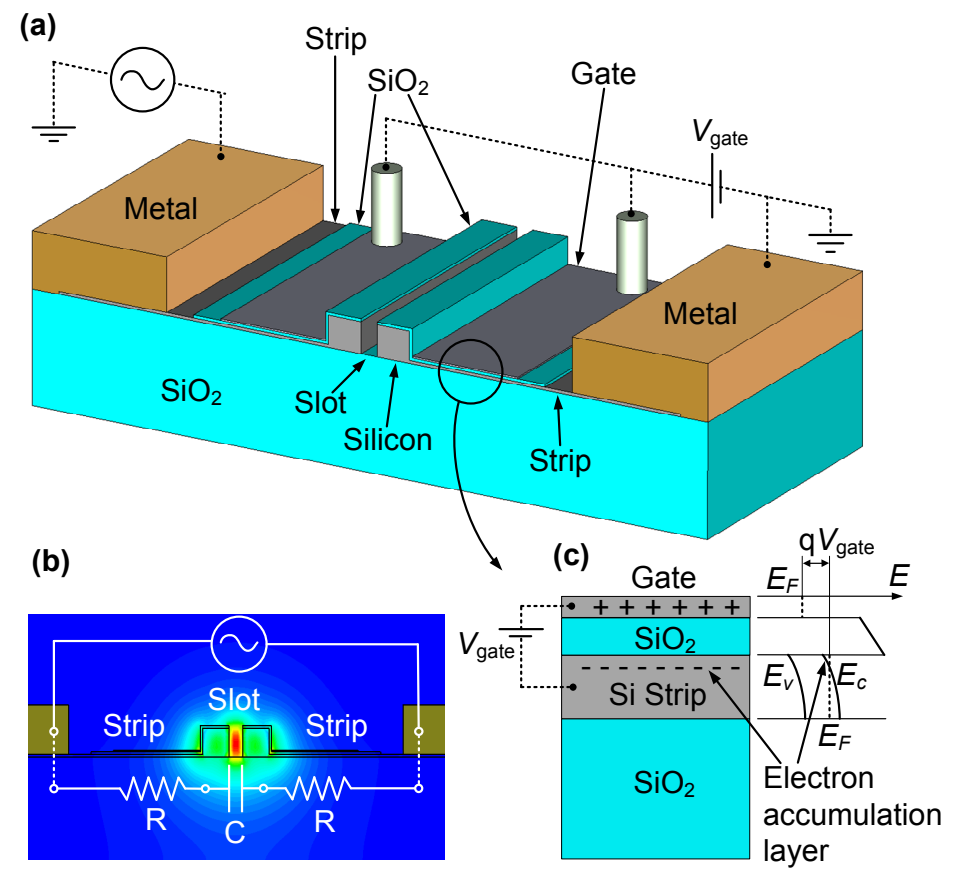

Figure 1. Example of SOH-modulator. (a) The optical active region (slot) is connected to the metal electrodes by means of thin silicon strips. On top of the silicon strips a $\mathrm{SiO}_{2}$ film is deposited and covered with the gate electrode ( $n$-type polysilicon). The entire waveguide is covered with a nonlinear organic cladding which uniformly fills the slot (not shown). (b) Cross-section of the waveguide and electric field distribution of the optical mode (quasi-TE, dominant electric field in parallel to the substrate plane); the light is concentrated in the slot. Lower inset: Equivalent RC circuit for the transfer of the voltage between metallic electrodes to the voltage dropping across the slot (slot capacitance C, strip resistance R). (c) When a positive gate voltage is applied across the gate oxide, a highly conductive electron accumulation layer is formed in the silicon strips. Under the influence of the gate voltage $V_{\text {gate }}$ the energy bands in the strip are bent. The quantities $E_{F, C, V}$ are Fermi energy, conduction and valence band edge energy, respectively; $q$ is the elementary charge. In the experiments reported in this work, the gate voltage is applied between the silicon strips and the silicon substrate instead [3].

Advanced modulation formats like QAM require, however, a linear response and pure phase modulation, making the exploitation of the electro-optic effect (Pockels effect [13]) particularly desirable.

The SOH platform is an ideal candidate for fulfilling these requirements [14-17]. In an SOH modulator, the optical quasi-TE field (dominant electric field in parallel to the substrate plane) is guided by a silicon waveguide, while the electro-optic effect is provided by an organic cladding with a high $\chi^{(2)}$-nonlinearity. The optical nonlinear interaction 
occurs inside a nanoscale slot, taking advantage of the field-enhancement caused by discontinuity of the refractive index. In these modulators, the optical nonlinear region needs to be connected to the modulating electrical source, Figure 1. This requires electrodes, which are both optically transparent and electrically highly conductive. To this end we exploit a thin silicon strip and a highly conductive electron accumulation layer which is induced by an external DC "gate" voltage. As opposed to doping, the electron mobility is not impaired by impurity scattering so that sheet resistances as low as $3 \mathrm{k} \Omega / \mathrm{sq}$ can be obtained with small optical loss [3]. This way we demonstrate for the first time an $\mathrm{SOH}$ electro-optic modulator having a $3 \mathrm{~dB}$ bandwidth larger than $90 \mathrm{GHz}$; this device has voltage-length product of $V_{\pi} L=11 \mathrm{~V} \mathrm{~mm}$ - the smallest ever reported value for a high-speed silicon-based modulator. Using a similar device in IQ-configuration (longer and with lower bandwidth), we demonstrate a data rate of $56 \mathrm{Gbit} / \mathrm{s}$ with a bit-error-ratio (BER) smaller than $10^{-10}$ when using an equalizer on the receiver.

\subsection{Over $90 \mathrm{GHz}$ bandwidth phase modulators}

High-speed electro-optic modulators continue attracting major attention from the integrated-optics community. However, even if a 3dB-bandwidth of $32 \mathrm{GHz}$ has been demonstrated in silicon-photonics already four years ago [18], no integrated device has been capable so far to approach the psychological threshold of $100 \mathrm{GHz}$ [12]. This is true not only for silicon-based devices (silicon notoriously suffers from small mobilities), but also for III-V materials like InP or $\mathrm{GaAs}$, or any other integrated technology available. Several reasons limit the device speed, comprising RC-limitations, carrier lifetime, and long and lossy electrode designs [9]. In the present work we utilize a $500 \mu \mathrm{m}$ long, highly-efficient nonlinear waveguide, together with a gate-induced highly-conductive charge accumulation layer for diminishing the RC time constant. The silicon waveguide has been fabricated at CEA-Leti and the silver metallization has been performed in-house.

(a)

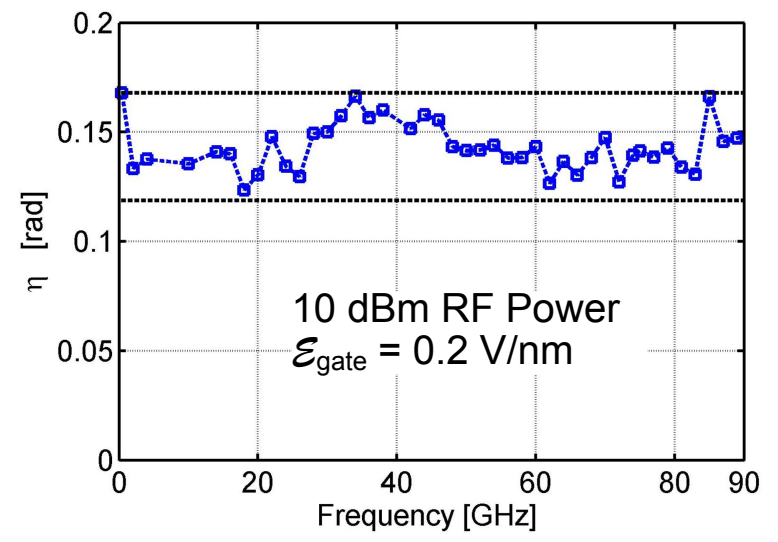

(b)

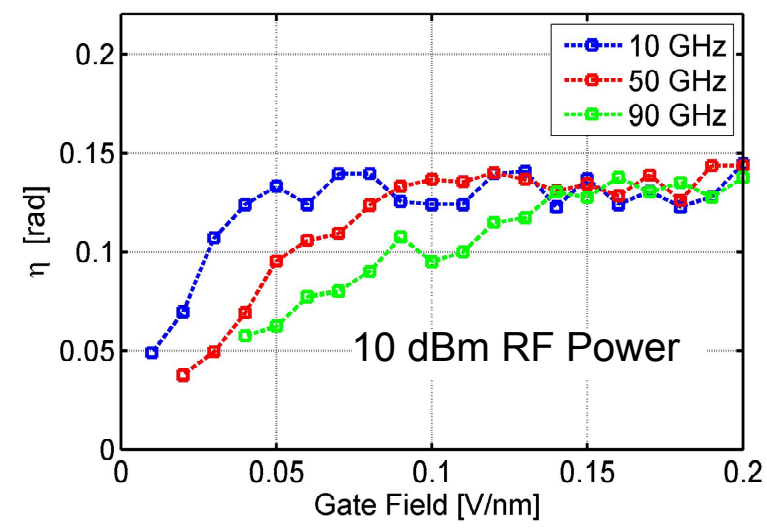

Figure 2. Modulation index $\eta$ of the DUT vs. frequency and gate field for $10 \mathrm{dBm}$ on-chip RF power (a) Phase modulation index $\eta$ vs. modulating frequency. The gate field is $0.2 \mathrm{~V} / \mathrm{nm}$; the gate voltage is applied across the $2 \mu \mathrm{m}$ silicon oxide substrate. The upper dotted black line represents the maximum of the modulation index $\eta$, the lower dotted horizontal stands for $70.7 \%$ of maximum ( $3 \mathrm{~dB}$ EOE bandwidth larger than $90 \mathrm{GHz}$ ). (b) Modulation index vs. gate field for selected modulation frequencies. Each curve reaches a plateau at high gate fields, showing that the RC-time constant does not limit the bandwidth of the device for high gate fields.

The device has been characterized by monitoring the modulation sidebands on an optical spectrum analyzer (OSA); this allows to calculate the modulation index, which is the maximum phase shift achieved in a cycle [3]. As electrical sources three independent RF sources and calibrated electrical power meters have been used. A calibration substrate (CS-8 from GGB Industries, Inc.) has been used for subtracting the loss of the RF cables and picoprobes. The modulation index normalized to $10 \mathrm{dBm}$ of RF power ( $1 \mathrm{~V}$ amplitude at $50 \Omega$ impedance) is shown in Figure 2(a). The minimum value of the modulation index in the entire measured range is larger than $70.7 \%$ of its maximum value, indicating that the $3 \mathrm{~dB}$ EO bandwidth of the modulator is larger than $90 \mathrm{GHz}$ (the modulation index is linearly proportional to the voltage and to 
the square root of the power). The modulation index has been monitored for the frequencies $10,50,90 \mathrm{GHz}$ while the gate field was increased from $0 \mathrm{~V} / \mathrm{nm}$ to $0.2 \mathrm{~V} / \mathrm{nm}$, Figure 2(b). As can be seen, each curve reaches a plateau at large gate fields, indicating that the RC time constant does not limit the device bandwidth for large gate fields.

Despite its large bandwidth, our device has a voltage-length product of $V_{\pi} L=11 \mathrm{Vmm}$ which very well competes with the best silicon-photonic modulators available today $[10,19,20]$.

\section{$2.256 \mathrm{Gbit} / \mathrm{s}$ QPSK modulation}

In order to show that $\mathrm{SOH}$ modulators can successfully support higher modulation formats, we combined four $\mathrm{SOH}$ phase-shifters into an IQ modulator as depicted in Figure 3(a). The phase shifters have a length of $1.5 \mathrm{~mm}$ and the entire chip, comprising the metal stack, has been fabricated in a CMOS fab (IMEC). The oxide over the waveguide and inside the slots has been removed by dry and wet etching. Low-loss mode converters have been used to excite the optical modes in the slot waveguide [21].

A $56 \mathrm{Gbit} / \mathrm{s}$ QPSK signal with $V_{\mathrm{pp}}=4.5 \mathrm{~V}$ was launched into the two ground-signal-ground (GSG) electrodes. Each Mach-Zehnder interferometer (MZI) was terminated with an external $50 \Omega$ resistor. Two bias-Ts were used for fine phase adjustments. The signal was then received with an optical modulation analyzer and equalized with a digital filter using 20 taps and having a length of 20 symbols.

The constellation diagram, as well as the eye diagrams for I and Q separately, can be seen in Figure 3. A bit-error-ratio of less than $10^{-10}$ is recorded when a gate field of $0.1 \mathrm{~V} / \mathrm{nm}$ was applied between the silicon substrate and the device silicon layer for increasing the device's bandwidth.

(a)
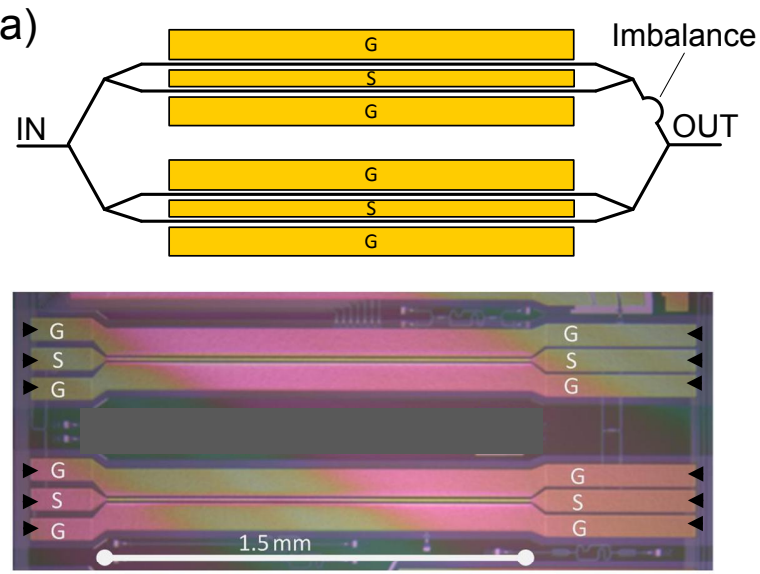

(b)

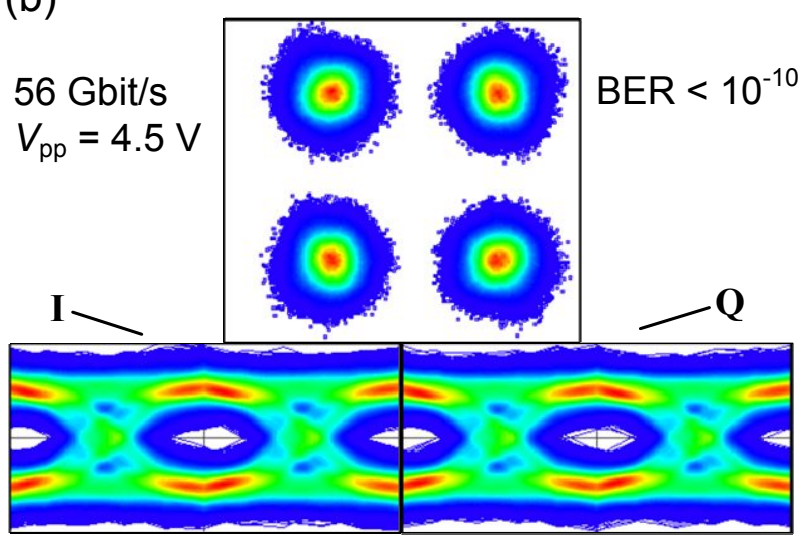

Figure 3. (a) Sketch of the modulator structure and optical microscope image of the fabricated IQ modulator. Two child Mach-Zehnder modulators (MZM) are combined in an imbalanced parent MachZehnder interferometer. The optical waveguides are partially located beneath the two ground-signalground (GSG) electrodes. The active region has a length of $1.5 \mathrm{~mm}$; the electrodes are longer for packaging reasons. (b) Constellation diagram at $56 \mathrm{Gbit} / \mathrm{s}$, single polarization, $V_{\mathrm{pp}}=4.5 \mathrm{~V}$. A bit-errorratio (BER) of less than $10^{-10}$ is recorded.

\section{LOW-VOLTAGE AND LOW-POWER SOH PHASE SHIFTER}

Phase shifters are essential for a large variety of applications in silicon photonics such as optical matrix switches, polarization controllers, tunable filters, and delay-interferometers, e. g., in fast Fourier transformers [22]. To enable dense integration, phase shifters must be compact, power-efficient, and tuning voltages should be below $1 \mathrm{~V}$. Thermal phase shifters exploit the high thermo-optic coefficient of silicon by heating silicon nanowires with a constant current flow. These devices enable phase shifts of few $\pi[23,24]$, however, their viability is limited by their power dissipation and the associated limitations in integration density.

Liquid crystals offer the potential of maintaining a phase shift with negligible power consumption [5, 25-27], but at present, low phase shifting efficiencies lead to long devices or high drive voltages. 
In this section we report on a phase shifter with a record-small voltage-length product of $V_{\pi} L=0.06 \mathrm{Vmm}$. This is obtained by combining liquid crystals with slot-waveguides within the silicon-organic hybrid platform [28].

\subsection{Design and Fabrication}

Liquid-crystals are highly birefringent materials, which can reorient under the influence of an electric field. This makes them ideal candidates for electro-optic applications. Strip-loaded slot waveguides enable efficient interaction of the guided light with a functional cladding, and at the same time allow generating high modulating electric field strengths [3]. The combination of these advantages is expected to lead to highly-efficient phase shifters with purely capacitive input impedances and therefore ultra-low power consumption.

Figure 4 depicts the strip-loaded slot waveguide used in the experiment. The external voltage is applied to the metallic electrodes located at a sufficient distance from the optical slot waveguide. The applied voltage drops entirely across the $120 \mathrm{~nm}$ wide slot, creating a strong electric field which can reorient the director of the liquid crystal deposited in the slot. The resistive silicon strips together with the capacitive slot act as an $R C$-circuit, whose time constant is negligible because of the slight doping of the silicon strips [3]. The phase shifter region is $1.7 \mathrm{~mm}$ long and has been fabricated entirely in the silicon fab of CEA-Leti, see Ref. [3] for a detailed description. The liquid crystal we tested is the commercially available E7 mixture, which is commonly used in liquid crystal displays.

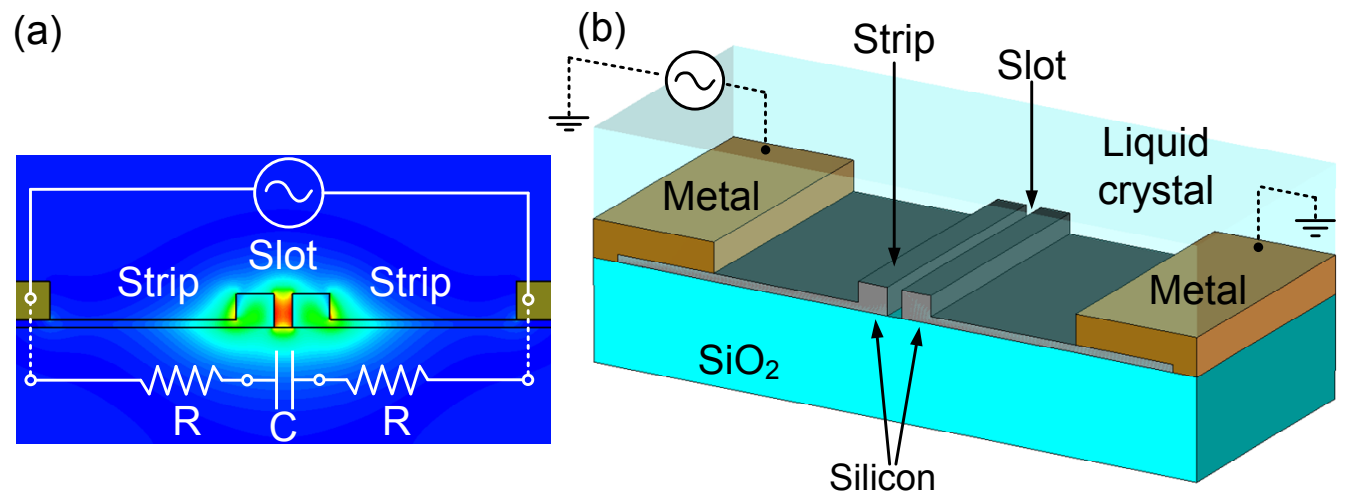

Figure 4. Strip-loaded slot waveguide. (a) Cross-section of the optical waveguide. The optical active region is connected to the metal electrodes by thin silicon strips (strip load). The color code represents the electric field magnitude of the optical mode as in Figure 1b; the light is concentrated inside the slot. Also indicated is the lumped equivalent circuit of the device. The slot is represented by a capacitance and the strip-load by a resistance. (b) Sketch of the waveguide. The liquid crystal is assumed to cover the waveguide homogeneously, and to entirely fill the slot. The nominal rail and slot widths of the waveguide are $120 \mathrm{~nm}$ and $240 \mathrm{~nm}$, respectively.

\subsection{Results}

In order to prevent ion migration in the liquid crystal, we applied a $100 \mathrm{kHz}$ electrical square wave, as common in LC technology. The liquid crystal orientation, and therefore the optical phase-shift, can then be tuned by varying the amplitude of the square wave. An example is to be seen in Figure 5, where the envelope of the $100 \mathrm{kHz}$ square signal is modulated with a $100 \mathrm{~Hz}$ triangular wave having $4 \mathrm{~V}$ amplitude. The maximum phase shift achieved in the $1.7 \mathrm{~mm}$ long device is as high as $80 \pi$ (251 rad), Figure 5. Additionally, we observed a voltage-dependent insertion loss (blue curve in Figure 5), which is attributed to the light scattering occurring at the liquid crystal domain boundaries [29, 30]. The maximum slope of the phase-shift response corresponds to a voltage-length product of $V_{\pi} L=0.06 \mathrm{~V} \mathrm{~mm}$, which is, to the best of our knowledge, the smallest value ever reported so far in a silicon-based device.

Since the capacitance of the device is about $0.1 \mathrm{pF}$, the energy needed for varying the voltage from $0 \mathrm{~V}$ to $4 \mathrm{~V}$ is less than $1 \mathrm{pJ}$. Repeating this process at the frequency of $100 \mathrm{kHz}$ would therefore lead to sub- $\mu \mathrm{W}$ power consumption. The power dissipated by the internal resistance of the device, which is about $20 \mathrm{G} \Omega$, is instead in the $\mathrm{nW}$ range. Because of the high phase shifting efficiency, a device length of $100 \mu \mathrm{m}$ is sufficient for most applications. In this case a $\pi$-shift could be obtained with a voltage swing smaller than $1 \mathrm{~V}$, and the voltage-dependent loss would be smaller than $0.3 \mathrm{~dB}$. 


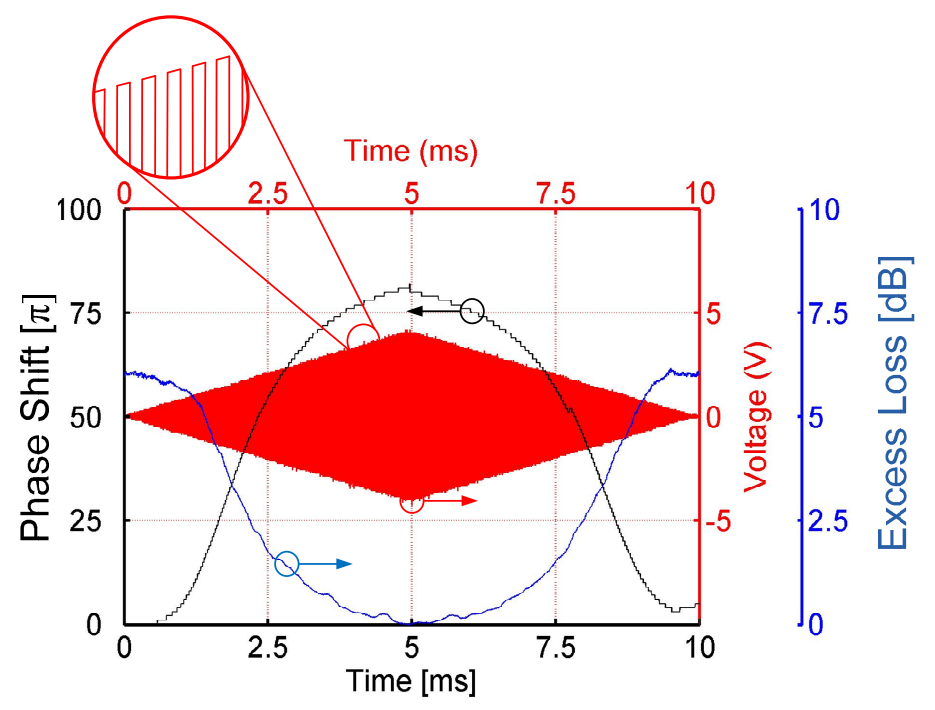

Figure 5. Liquid crystal phase shifter. Phase shift, applied voltage and excess optical loss vs. time. The envelope of a $100 \mathrm{kHz}$ square wave is modulated with a triangular function having frequency $100 \mathrm{~Hz}$. The maximum electrical amplitude is $4 \mathrm{~V}$. The maximum phase shift achieved is about $80 \pi$. The device length is $1.7 \mathrm{~mm}$.

\section{DIFFERENCE-FREQUENCY GENERATION (DFG)}

Difference-frequency generation and other second-order nonlinear processes such as spontaneous down-conversion or optical parametric amplification (OPA) are essential for a number of applications, ranging from spectroscopy, free-space communication, biochemical sensing, medical therapy, ultra-fast optical signal processing, lowest-noise optical amplification, and quantum physics. Since at least one of the frequencies involved in a three-wave mixing process is necessarily well separated from the others, second-order processes represent in a natural way an excellent candidate for generating mid-IR and far-IR wavelengths [31].

Efficient nonlinear conversion require materials with strong nonlinearities, high-optical intensities, and phase-matching between the waves involved [13]. So far, second-order nonlinear waveguides have been made of polymers, GaAs, InP, and $\mathrm{LiNbO}_{3}$ [32], and phase-matching has successfully been achieved by birefringence, intermodal dispersion, or quasiphase matching. These materials often require specialized process technologies which are not applicable for mass production. Further, the relatively small index contrasts which can be achieved limit the modal confinement in waveguides. Hence, conversion efficiencies are small, and optical output powers are usually only in the $\mu \mathrm{W}$ range [32].

The widely available silicon photonic technology, on the other hand, allows fabricating high-index contrast waveguides for obtaining the required intensities with low optical powers. The vision of creating mid-IR applications using the inexpensive silicon-photonics platform [33] has already led to a number of publications on the following topics: Silicon waveguides pumped below the two-photon absorption (TPA) edge with powers as high as $33.5 \mathrm{~W}$ (45 $\mathrm{dBm})$ [34], lowloss propagation in the 2-6 $\mu \mathrm{m}$ wavelength range [35, 36], light generation at $2.4 \mu \mathrm{m}$ with standard telecom sources [37], high-Q SOI photonic crystal cavities at $4.4 \mu \mathrm{m}$ [38], Raman amplification at $3.39 \mu \mathrm{m}$ [39], and extensive simulations for single-mode operation and polarization-independent operation in SOI rib waveguides in the mid-IR region [40].

However, unstrained crystalline silicon is centrosymmetric, and its second-order nonlinearity is zero. As a consequence, mid-IR generation in unstrained silicon waveguides has to rely on silicon's third-order nonlinearity [34], taking advantage of the "built-in" strong Kerr nonlinearity of crystalline [41] or amorphous silicon [42]. This, in turn, results in high pump power requirements.

In silicon photonics, first attempts of second-harmonic generation are based on strained waveguides, but despite nanosecond peak pump powers as high as $0.7 \mathrm{~W}$, the output peak powers are limited to only $40 \mathrm{nW}$ [43]. The small efficiency is due to a non-phase-matched design. A method for achieving quasi-phase matching (QPM) in periodically strained silicon has already been proposed [44], however, large mode sizes and small nonlinearities lead to normalized 
conversion efficiencies smaller than $1 \% \mathrm{~W}^{-1} \mathrm{~cm}^{-2}$. More recently, a method of achieving phase-matching based on birefringence in strained silicon waveguides has been proposed [45], but the efficiency of the device relies on nonlinearities which have not been shown so far in waveguides of the proposed size [46]. Finally, the potentially high efficiencies of $\mathrm{SOH}$ second-order nonlinear waveguides have already been discussed [14], but unfortunately no waveguide design has been proposed so far [47].

\subsection{The proposed second-order nonlinear device}

In order to obtain phase-matching in a $\mathrm{SOH}$ waveguide, we propose a double slot waveguide, Figure 6 . It consists of three parallel silicon strips realized on standard silicon-on-insulator (SOI) wafer having an oxide thickness of $2 \mu \mathrm{m}$ and a device layer of $220 \mathrm{~nm}$. The waveguide is spin coated with polymer-dispersed nonlinear chromophores [48], which are processed to have a high $\chi^{(2)}$-nonlinearity only inside the two slots. This can be experimentally achieved by poling [3] the material at its glass transition temperature, and by applying two voltages as shown in Figure 6.

For definitiveness, we will describe a device for difference-frequency generation (DFG) with a pump wavelength of $1.5 \mu \mathrm{m}$, a signal wavelength of $2.9 \mu \mathrm{m}$ and an idler wavelength close to $3.1 \mu \mathrm{m}$. Signal and idler propagate in a mode different from the mode in which the pump light is guided. The concept, however, is more general and may be applied to other spectral ranges as will be outlined below.

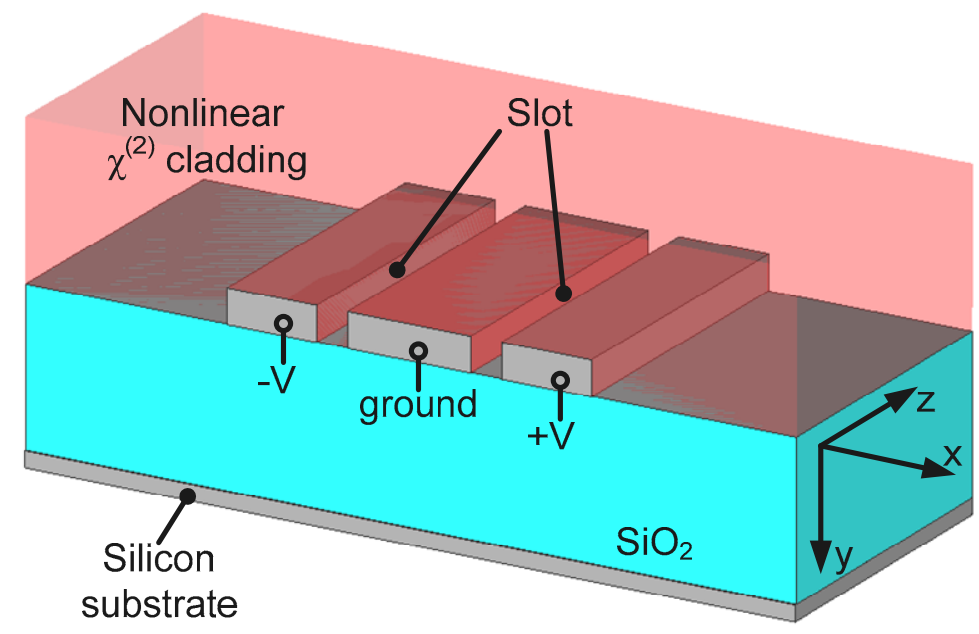

Figure 6. Silicon organic-hybrid (SOH) double slot waveguide for second-order nonlinear applications. The waveguide consists of three silicon strips on a glass substrate, it is multimoded and dimensioned such that modal phase-matching is achieved. The waveguide is covered by a nonlinear cladding, which is poled during fabrication by applying the voltages $-\mathrm{V}$ and $+\mathrm{V}$ to the outermost strips while the central strip is grounded. As a result, the nonlinear second-order susceptibility is high only inside the slots.

\subsection{Phase-matching}

The waveguide is dimensioned such that modal phase-matching (MPM) is achieved [32] between the quasi-TE $\mathrm{T}_{40}$ mode for the pump (four nodes in the horizontal direction, zero nodes in the vertical direction) and the fundamental quasi- $\mathrm{TE}_{00}$ mode for signal and idler. These modes can be excited efficiently with special mode converters [47].

By a thorough numerical simulation of the dispersion of the optical modes involved, we find that for a fixed pump frequency and certain waveguide dimensions a pair of signal and idler frequencies exist satisfying the phase-matching condition. This information is represented in Figure 7. The three curves stand for three different waveguide geometries, that can be chosen by the user according to the desired pump and signal wavelengths. The cyan regions show the frequencies for which the coherent buildup length [13] is equal or larger than $1 \mathrm{~cm}$. Therefore a wavelength detuning of $50 \mathrm{~nm}$ or more from the ideal operating points still allows a coherent buildup of the converted wave, showing that the wavelength tuning requirements are very relaxed. 


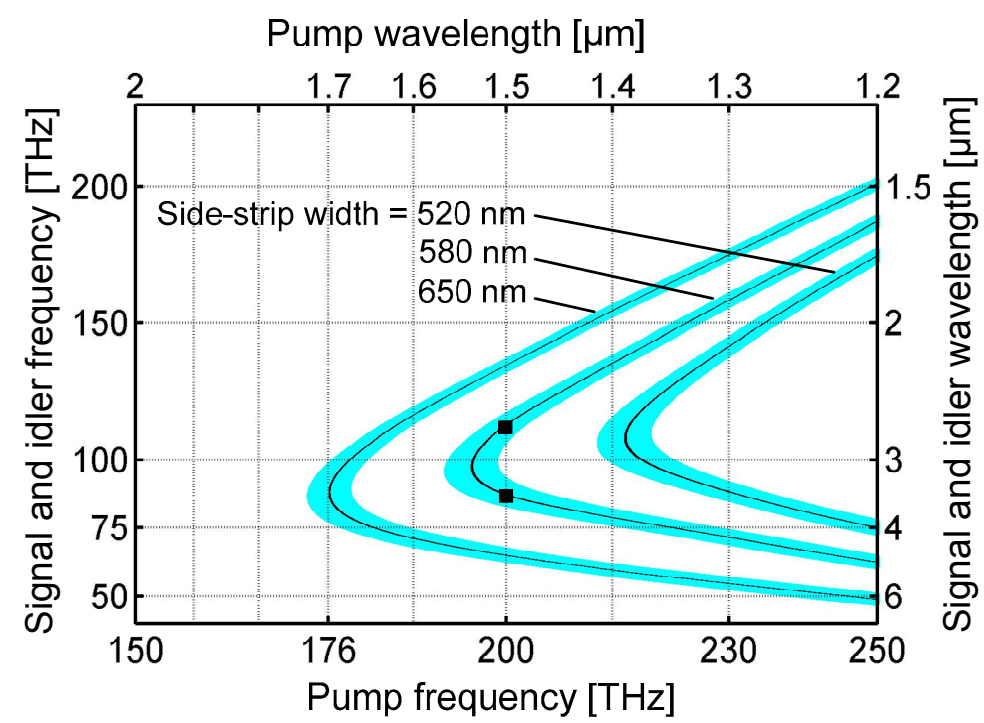

Figure 7. Signal and idler frequencies vs. pump frequency for three different geometries. The black curves specify for a given pump frequency the signal and idler frequencies which satisfy the energy conservation and the phase-matching conditions. The cyan-coloured regions indicate the frequency space where the coherent buildup length $L_{\mathrm{coh}}=2 /\left(k_{s}+k_{i}-k_{p}\right)$ [13] is equal to $1 \mathrm{~cm}$ or longer. The three different curves represent waveguides where the side-strip width is set to $520 \mathrm{~nm}, 580 \mathrm{~nm}$ and $650 \mathrm{~nm}$. The central-strip width is $800 \mathrm{~nm}$ and the slot width is $200 \mathrm{~nm}$ in all the three cases. For a side-strip width of e.g. $580 \mathrm{~nm}$ and a pump wavelength of $1.5 \mu \mathrm{m}(200 \mathrm{THz})$, signal and idler wavelengths of $2.6 \mu \mathrm{m}$ and $3.5 \mu \mathrm{m}$ would result (square symbols).

\subsection{Power levels and conversion efficiencies}

A convenient quantity [32] for representing the device performance is the normalized conversion efficiency, which is defined by

$$
\eta=\lim _{z \rightarrow 0} P_{i}(z) /\left(P_{s}(0) P_{p}(0) z^{2}\right)
$$

where $P_{i, s, p}(z)$ is the power of signal, idler and pump lightwaves at position $z$. We calculated numerically this quantity by performing a suited overlap integral between the optical modes involved and the nonlinear susceptibility tensor [47]. For the latter we assumed the value $\left|\chi_{111}\right|=230 \mathrm{pm} / \mathrm{V}$, which corresponds to an electro-optic coefficient $r_{33}=70 \mathrm{pm} / \mathrm{V}$ [3]. Propagation losses are neglected.

As a result we find a normalized conversion efficiency as high as

$$
\eta=29 \mathrm{~W}^{-1} \mathrm{~cm}^{-2} \quad\left(2900 \% \mathrm{~W}^{-1} \mathrm{~cm}^{-2}\right)
$$

As an example, assuming a $\mathrm{CW}$ pump power of $20 \mathrm{dBm}$, the calculated conversion efficiency corresponds to a signal and idler power gain equal to $14.7 \mathrm{~dB} / \mathrm{cm}$ (in the limit of long device length). As a second example, assuming $20 \mathrm{dBm}$ $\mathrm{CW}$ input pump power, $-10 \mathrm{dBm}$ signal input power, no idler at the input and neglecting losses, after propagating through a $1 \mathrm{~cm}$ long waveguide the idler has a power of $0.68 \mathrm{~mW}(-1.7 \mathrm{dBm})$, and the signal has a power of $0.78 \mathrm{~mW}$ $(-1.1 \mathrm{dBm})$.

We observe that the assumed nonlinear susceptibility of $230 \mathrm{pm} / \mathrm{V}$ is a very conservative value. In fact, nonlinear susceptibilities of $354 \mathrm{pm} / \mathrm{V}$ have already been measured at optical frequencies in nonlinear polymers [49], and this value increases to $580 \mathrm{pm} / \mathrm{V}$ for organic crystals [50]. 


\subsection{Third-order nonlinearity vs. second-order nonlinearity}

We further compare third-order with second-order nonlinear devices for state-of-the-art nonlinear materials, finding that second-order waveguides can be far more efficient than any other third-order nonlinear device. To this end, we calculate the electric field strength required for creating a certain nonlinear polarization. The optical response of a material can be described by expanding the polarization $P(t)$ as a power series of the electric field strength $E(t)$. For simplicity we represent the vector fields $P$ and $E$ by scalar quantities,

$$
P(t)=P^{(1)}(t)+P^{(2)}(t)+P^{(3)}(t)+\cdots, \quad P^{(q)}=\chi^{(q)} E^{q} .
$$

The second-order polarization $P^{(2)}$ is always larger than the third-order polarization $P^{(3)}$ if the electric field is smaller than the critical field

$$
E_{\mathrm{c}}=\frac{\chi^{(2)}}{\chi^{(3)}} \text {. }
$$

If we now substitute $\chi^{(2)}=230 \mathrm{pm} / \mathrm{V}$ and $\chi^{(3)}=10^{5} \mathrm{pm}^{2} / \mathrm{V}^{2}$ (this value corresponds to the third-order nonlinear organic molecule DDMEBT which has previously been used for frequency conversion in SOI slot waveguides [4] and has one of the highest Kerr nonlinearities at $1.5 \mu \mathrm{m}$ [4]), we find that the critical electric field is $E_{\mathrm{c}}=2.310^{9} \mathrm{~V} / \mathrm{m}$.

For the hypothetical case of a plane wave in vacuum, this field corresponds to an (enormous) intensity of $I=\varepsilon_{0} c|E|^{2}=1.410^{16} \mathrm{~W} / \mathrm{m}^{2}$, or $140 \mathrm{~W}$ on an area of $100 \times 100 \mathrm{~nm}^{2}$. Since practical devices operate at intensities significantly smaller than the latter [34], $\chi^{(2)}$ waveguides will be more efficient than their $\chi^{(3)}$ counterparts.

\section{SUMMARY}

We report recent progresses in silicon-organic hybrid $(\mathrm{SOH})$ high-speed modulators, low-power liquid-crystal phase shifters and second-order nonlinear waveguides. We demonstrate a polymer-clad phase shifter having a $3 \mathrm{~dB}$ bandwidth larger than $90 \mathrm{GHz}$, which is, to the best of our knowledge, the fastest silicon-based modulator demonstrated so far. Despite its large bandwidth, our device has a voltage-length product of $V_{\pi} L=11 \mathrm{Vmm}$ which very well competes with the best silicon-photonic modulators available today.

We further show that liquid crystals in a silicon slot waveguides can be efficiently driven by AC electric fields, solving the issue of ion-drift. We demonstrate a record-low voltage-length product of $V_{\pi} L=0.06 \mathrm{Vmm}$, and sub- $\mu \mathrm{W}$ power consumption.

Finally, we propose a silicon waveguide concept suited for three-wave mixing. We show that the necessary phasematching is possible in a silicon-organic hybrid (SOH) waveguide. This could be achieved by dispersion engineering. Our device has high conversion efficiency, is based on standard materials and technologies, and allows all-optical signal processing, mid-IR generation, and lowest-noise optical parametric amplification with small optical pump powers.

\section{ACKNOWLEDGMENTS}

We acknowledge support by the DFG Center for Functional Nanostructures (CFN), the KIT Initiative of Excellence, the Karlsruhe School of Optics and Photonics (KSOP), the EU-FP7 projects SOFI (grant 248609) and EURO-FOS (grant 224402), the DFG Center for Functional Nanostructures (CFN), and by the BMBF joint project MISTRAL, funded by the German Ministry of Education and Research under grant 01BL0804. We further acknowledge technological support by the Karlsruhe Nano-Micro Facility (KNMF). We are grateful for technological support by the Light Technology Institute (KIT-LTI), CEA-Leti fab within ePIXfab (grant 224232) and the ePIXfab (silicon photonics platform). We acknowledge the Helmholtz International Research School for Teratronics at KIT, and the BMBF joint project MISTRAL, funded by the German Ministry of Education and Research under grant 01BL0804. 


\section{REFERENCES}

[1] L. Kimerling, "Scaling Information Technology on the Silicon Platform," European Conference on Optical Communication (ECOC 2010), paper Tu.4.C.1 (2010).

[2] R. Ding, T. Baehr-Jones, Y. Liu, R. Bojko, J. Witzens, S. Huang, J. Luo, S. Benight, P. Sullivan, J. M. Fedeli, M. Fournier, L. Dalton, A. Jen, and M. Hochberg, "Demonstration of a low V pi L modulator with GHz bandwidth based on electro-optic polymer-clad silicon slot waveguides," Optics Express 18, 15618-15623 (2010).

[3] L. Alloatti, D. Korn, R. Palmer, D. Hillerkuss, J. Li, A. Barklund, R. Dinu, J. Wieland, M. Fournier, J. Fedeli, H. Yu, W. Bogaerts, P. Dumon, R. Baets, C. Koos, W. Freude, and J. Leuthold, "42.7 Gbit/s electro-optic modulator in silicon technology," Optics Express 19, 11841-11851 (2011).

[4] C. Koos, P. Vorreau, T. Vallaitis, P. Dumon, W. Bogaerts, R. Baets, B. Esembeson, I. Biaggio, T. Michinobu, F. Diederich, W. Freude, and J. Leuthold, "All-optical high-speed signal processing with silicon-organic hybrid slot waveguides," Nature Photonics 3, 216-219 (2009).

[5] W. D. Cort, J. Beeckman, R. James, F. A. Fernandez, R. Baets, and K. Neyts, "Tuning silicon-on-insulator ring resonators with in-plane switching liquid crystals," J. Opt. Soc. Am. B 28, 79-85 (2011).

[6] J. Pfeifle, L. Alloatti, W. Freude, J. Leuthold, and C. Koos, "Silicon-organic hybrid phase shifter based on a slot waveguide with a liquid-crystal cladding," Optics Express 20, 15359-15376 (2012).

[7] M. Lauermann, D. Korn, P. Appel, L. Alloatti, W. Freude, J. Leuthold, and C. Koos, "Silicon-organic hybrid (SOH) lasers at telecommunication wavelengths," Conf. on Integrated Photonics Research, Silicon and NanoPhotonics (IPR'12), Paper IM3A.3 (2012).

[8] W. M. J. Green, M. J. Rooks, L. Sekaric, and Y. A. Vlasov, "Ultra-compact, low RF power, 10 gb/s silicon Mach-Zehnder modulator," Optics Express 15, 17106-17113 (2007).

[9] L. Liao, A. Liu, D. Rubin, J. Basak, Y. Chetrit, H. Nguyen, R. Cohen, N. Izhaky, and M. Paniccia, "40 Gbit/s silicon optical modulator for high-speed applications," Electronics Letters 43, 1196-1197 (2007).

[10] T. Baehr-Jones, R. Ding, Y. Liu, A. Ayazi, T. Pinguet, N. C. Harris, M. Streshinsky, P. Lee, Y. Zhang, A. E. J. Lim, T. Y. Liow, S. H. G. Teo, G. Q. Lo, and M. Hochberg, "Ultralow drive voltage silicon traveling-wave modulator," Optics Express 20, 12014-12020 (2012).

[11] D. J. Thomson, F. Y. Gardes, J. M. Fedeli, S. Zlatanovic, Y. F. Hu, B. P. P. Kuo, E. Myslivets, N. Alic, S. Radic, G. Z. Mashanovich, and G. T. Reed, "50-Gb/s Silicon Optical Modulator," IEEE Photonics Technology Letters 24(2012).

[12] Y. B. Tang, J. D. Peters, and J. E. Bowers, "Over $67 \mathrm{GHz}$ bandwidth hybrid silicon electroabsorption modulator with asymmetric segmented electrode for 1.3 mu m transmission," Optics Express 20, 11529-11535 (2012).

[13] R. W. Boyd, Nonlinear Optics (Academic press, 2008).

[14] T. W. Baehr-Jones and M. J. Hochberg, "Polymer silicon hybrid systems: A platform for practical nonlinear optics," Journal of Physical Chemistry C 112, 8085-8090 (2008).

[15] J. M. Brosi, C. Koos, L. C. Andreani, M. Waldow, J. Leuthold, and W. Freude, "High-speed low-voltage electro-optic modulator with a polymer-infiltrated silicon photonic crystal waveguide," Optics Express 16, 4177-4191 (2008).

[16] M. Hochberg, T. Baehr-Jones, G. Wang, J. Huang, P. Sullivan, L. Dalton, and A. Scherer, "Towards a millivolt optical modulator with nano-slot waveguides," Optics Express 15, 8401-8410 (2007).

[17] J. H. Wülbern, A. Petrov, and M. Eich, "Electro-optical modulator in a polymer-infiltrated silicon slotted photonic crystal waveguide heterostructure resonator," Optics Express 17, 304-313 (2009).

[18] A. Liu, L. Liao, D. Rubin, J. Basak, Y. Chetrit, H. Nguyen, R. Cohen, N. Izhaky, and M. Paniccia, "Recent development in a high-speed silicon optical modulator based on reverse-biased pn diode in a silicon waveguide," Semiconductor Science and Technology 23, 064001-064001 (2008).

[19] J. F. Ding, H. T. Chen, L. Yang, L. Zhang, R. Q. Ji, Y. H. Tian, W. W. Zhu, Y. Y. Lu, P. Zhou, R. Min, and M. B. Yu, "Ultra-low-power carrier-depletion Mach-Zehnder silicon optical modulator," Optics Express 20, 70817087 (2012).

[20] H. Yu, M. Pantouvaki, J. Van Campenhout, D. Korn, K. Komorowska, P. Dumon, Y. L. Li, P. Verheyen, P. Absil, L. Alloatti, D. Hillerkuss, J. Leuthold, R. Baets, and W. Bogaerts, "Performance tradeoff between lateral and interdigitated doping patterns for high speed carrier-depletion based silicon modulators," Optics Express 20, 12926-12938 (2012). 
[21] R. Palmer, L. Alloatti, D. Korn, W. Heni, P. Schindler, J. Bolten, M. Karl, M. Waldow, T. Wahlbrink, W. Freude, C. Koos, and J. Leuthold, "Highly Efficient Strip-to-Slot Mode Converters," CLEO 2012, Paper CM4M1 (2012).

[22] D. Hillerkuss, M. Winter, M. Teschke, A. Marculescu, J. Li, G. Sigurdsson, K. Worms, S. Ben Ezra, N. Narkiss, W. Freude, and J. Leuthold, "Simple all-optical FFT scheme enabling Tbit/s real-time signal processing," Optics Express 18, 9324-9340 (2010).

[23] C. R. Doerr and L. Chen, "Monolithic PDM-DQPSK receiver in silicon," Proceedings of European Conference on Optical Communication (ECOC), Paper PD3_6 (2010).

[24] S. S. Djordjevic, L. W. Luo, S. Ibrahim, N. K. Fontaine, C. B. Poitras, B. Guan, L. Zhou, K. Okamoto, Z. Ding, M. Lipson, and S. J. B. Yoo, "Fully Reconfigurable Silicon Photonic Lattice Filters With Four Cascaded Unit Cells," Photonics Technology Letters, IEEE 23, $42-44$ (2011).

[25] N. Hattasan, W. De Cort, J. Beeckman, K. Neyts, and R. Baets, "Tunable Silicon-on-Insulator based integrated optical filters with liquid crystal cladding," in LEOS Annual Meeting Conference Proceedings, 2009. LEOS '09. IEEE, 189-190.

[26] J. Li, S. T. Wu, S. Brugioni, R. Meucci, and S. Faetti, "Infrared refractive indices of liquid crystals," Journal of Applied Physics 97, 073501-073501 (2005).

[27] H. Desmet, W. Bogaerts, A. Adamski, J. Beeckman, K. Neyts, and R. Baets, "Silicon-on-insulator optical waveguides with liquid crystal cladding for switching and tuning," in Proceedings of European Conference on Optical Communication, 430-431.

[28] J. Leuthold, W. Freude, J. M. Brosi, R. Baets, P. Dumon, I. Biaggio, M. L. Scimeca, F. Diederich, B. Frank, and C. Koos, "Silicon Organic Hybrid Technology-A Platform for Practical Nonlinear Optics," Proc. IEEE 97, 1304-1316 (2009).

[29] Y. H. Fan, Y. H. Lin, H. W. Ren, S. Gauza, and S. T. Wu, "Fast-response and scattering-free polymer network liquid crystals for infrared light modulators," Applied Physics Letters 84, 1233-1235 (2004).

[30] M. Kobayashi, H. Terui, M. Kawachi, and J. Noda, "2x2 Optical-waveguide Matrix Switch Using Nematic Liquid-crystal," IEEE Journal of Quantum Electronics 18, 1603-1610 (1982).

[31] A. B. Sugiharto, C. M. Johnson, H. B. De Aguiar, L. Alloatti, and S. Roke, "Generation and application of high power femtosecond pulses in the vibrational fingerprint region," Applied Physics B-lasers and Optics 91, 315318 (2008).

[32] S. V. Rao, K. Moutzouris, and M. Ebrahimzadeh, "Nonlinear frequency conversion in semiconductor optical waveguides using birefringent, modal and quasi-phase-matching techniques," Journal of Optics A-pure and Applied Optics 6, 569-584 (2004).

[33] R. Soref, "Mid-infrared photonics in silicon and germanium," Nature Photonics 4, 495-497 (2010).

[34] X. P. Liu, J. B. Driscoll, J. I. Dadap, R. M. Osgood, S. Assefa, Y. A. Vlasov, and W. M. J. Green, "Self-phase modulation and nonlinear loss in silicon nanophotonic wires near the mid-infrared two-photon absorption edge," Optics Express 19, 7778-7789 (2011).

[35] A. Spott, Y. Liu, T. Baehr-Jones, R. Ilic, and M. Hochberg, "Silicon waveguides and ring resonators at $5.5 \mathrm{mu}$ m," Applied Physics Letters 97, 213501-213501 (2010).

[36] F. X. Li, S. D. Jackson, C. Grillet, E. Magi, D. Hudson, S. J. Madden, Y. Moghe, C. O'Brien, A. Read, S. G. Duvall, P. Atanackovic, B. J. Eggleton, and D. J. Moss, "Low propagation loss silicon-on-sapphire waveguides for the mid-infrared," Optics Express 19, 15212-15220 (2011).

[37] S. Zlatanovic, J. S. Park, S. Moro, J. M. C. Boggio, I. B. Divliansky, N. Alic, S. Mookherjea, and S. Radic, "Mid-infrared wavelength conversion in silicon waveguides using ultracompact telecom-band-derived pump source RID D-4869-2011," Nature Photonics 4, 561-564 (2010).

[38] R. Shankar, R. Leijssen, I. Bulu, and M. Loncar, "Mid-infrared photonic crystal cavities in silicon," Optics Express 19, 5579-5586 (2011).

[39] V. Raghunathan, D. Borlaug, R. R. Rice, and B. Jalali, "Demonstration of a mid-infrared silicon Raman amplifier," Optics Express 15, 14355-14362 (2007).

[40] M. M. Milosevic, P. S. Matavulj, P. Y. Y. Yang, A. Bagolini, and G. Z. Mashanovich, "Rib waveguides for mid-infrared silicon photonics," Journal of the Optical Society Of America B-Optical Physics 26, 1760-1766 (2009).

[41] M. A. Foster, A. C. Turner, J. E. Sharping, B. S. Schmidt, M. Lipson, and A. L. Gaeta, "Broad-band optical parametric gain on a silicon photonic chip," Nature 441, 960-963 (2006). 
[42] B. Kuyken, H. Ji, S. Clemmen, S. K. Selvaraja, H. Hu, M. Pu, M. Galili, P. Jeppesen, G. Morthier, S. Massar, L. K. Oxenlowe, G. Roelkens, and R. Baets, "Nonlinear properties of and nonlinear processing in hydrogenated amorphous silicon waveguides," Optics Express 19, B146-153 (2011).

[43] M. Cazzanelli, F. Bianco, E. Borga, G. Pucker, M. Ghulinyan, E. Degoli, E. Luppi, V. Veniard, S. Ossicini, D. Modotto, S. Wabnitz, R. Pierobon, and L. Pavesi, "Second-harmonic generation in silicon waveguides strained by silicon nitride," Nature Materials 11, 148-154 (2012).

[44] N. K. Hon, K. K. Tsia, D. R. Solli, and B. Jalali, "Periodically poled silicon," Applied Physics Letters 94, 091116-091116 (2009).

[45] I. Avrutsky and R. Soref, "Phase-matched sum frequency generation in strained silicon waveguides using their second-order nonlinear optical susceptibility," Optics Express 19, 21707-21716 (2011).

[46] B. Chmielak, M. Waldow, C. Matheisen, C. Ripperda, J. Bolten, T. Wahlbrink, M. Nagel, F. Merget, and H. Kurz, "Pockels effect based fully integrated, strained silicon electro-optic modulator," Optics Express 19, 17212-17219 (2011).

[47] L. Alloatti, D. Korn, C. Weimann, C. Koos, W. Freude, and J. Leuthold, "Second-order nonlinear siliconorganic hybrid waveguides," Optics Express 20, 20506-20515 (2012).

[48] GigOptix, "www.gigoptix.com."

[49] A. D. Bristow, N. Rotenberg, and H. M. van Driel, "Two-photon absorption and Kerr coefficients of silicon for 850-2200 nm," Applied Physics Letters 90, 191104-191104 (2007).

[50] M. Jazbinsek, L. Mutter, and P. Gunter, "Photonic Applications With the Organic Nonlinear Optical Crystal DAST," IEEE Journal of selected topics in quantum electronics 14, 1298-1311 (2008). 